\title{
Optimised Process for Fabricating Tapered Long Period Gratings
}

\author{
K. Mullaney, S. E. Staines, S. W. James* and R. P. Tatam, \\ Engineering Photonics, \\ Cranfield University, Bedford, MK43 0AL, UK.
}

\begin{abstract}
The process of fabricating tapered long period gratings (TLPGs) using a $\mathrm{CO}_{2}$ laser is described. TLPGs with a period spacing of $378 \mu \mathrm{m}$, were fabricated by optimization of the taper waist diameter and careful control of the duty-cycle and its uniformity along the length of the grating. The 6-period TLPGs exhibited a pass-band insertion loss of $0.6 \mathrm{~dB}$, resonance band extinction values of $3 \mathrm{~dB}$ and had a physical length of $2.27 \mathrm{~mm}$.
\end{abstract}

Keywords: Optical fibre taper, $\mathrm{CO}_{2}$ laser, long period grating, laser material processing.

\section{INTRODUCTION}

Optical fibre long period gratings (LPGs) were developed as devices for optical communications ${ }^{1}$, where they have been used as band rejection filters, gain equalizers and wavelength tuning elements in fibre lasers ${ }^{2}$. They have also found many applications in the field of optical sensing ${ }^{3}$. LPGs can be fabricated by either UV irradiation, or the periodic application of heat over a sub-millimeter length of the fibre. The necessary heat source can be either a $\mathrm{CO}_{2} l a s e{ }^{4}$, arcdischarge ${ }^{5}$ or by resistive heating ${ }^{6}$. The use of $\mathrm{CO}_{2}$ laser irradiation is attractive, as its use is flexible, is repeatable and produces less contamination on the fibre surface compared to the alternative techniques ${ }^{4}$.

In tapered long period gratings (TLPGs), the fibre geometry is perturbed by periodically tapering the fibre. This is usually achieved by locally heating and stretching the fibre. In previous reports of this type of device, the taper waist diameter was typically between $70 \mu \mathrm{m}$ and $120 \mu \mathrm{m}{ }^{4,7}$. One of the key advantages of thermally induced gratings is their ability to retain their spectral properties at higher temperatures, as compared to UV written gratings, which start to degrade at temperatures in excess of $200{ }^{0} \mathrm{C}^{8}$. Thermally formed gratings typically start to degrade at temperatures close to the melting temperature of the fibre $\sim 1000{ }^{0} \mathrm{C}^{8}$. Another advantage of thermally generated gratings is that larger mode coupling coefficients can be achieved, compared to UV-written gratings, as the magnitude of the core index modulation is greater ${ }^{8}$. Spectral resonance bands of a given extinction coefficient can therefore be produced with fewer grating periods and so the overall physical length of the grating can be much shorter than that of a UV-written LPG with a comparable transmission spectrum.

A review of thermal approaches to LPG fabrication shows that high quality LPGs with well-defined resonance bands and low insertion losses have been fabricated either using the index modulation of the fibre using a $\mathrm{CO}_{2}$ laser (without tapering) ${ }^{9,10}$, or using arc discharge heating to both index modulate and geometrically taper the fibre ${ }^{5,11}$. The use of $\mathrm{CO}_{2}$ laser irradiation allows TLPGs to be fabricated with periods shorter ${ }^{12}$ than can be obtained with arc-discharge techniques. The dimensions of the electrodes in fusion splicers currently limits the minimum achievable grating period to $\sim 220 \mu \mathrm{m}^{11}$.

The aim of this work is to develop the $\mathrm{CO}_{2}$ laser-based fabrication of TLPGs so that their spectral characteristics are comparable with those fabricated using the arc-discharge approach, which typically exhibit insertion losses below $\sim 2 \mathrm{~dB}^{5,11}$. The losses which are currently observed with $\mathrm{CO}_{2}$ fabricated TLPGs are $>10 \mathrm{~dB}^{4,7}$. Optimisation of the taper geometry and grating duty cycle is shown to reduce optical insertion losses to below $\sim 2 \mathrm{~dB}$.

*s.w.james@cranfield.ac.uk ; phone +441234754623; http://www.cranfield.ac.uk

This paper was published in 25th International Conference on Optical Fiber Sensors and is made available with permission of SPIE. One print or electronic copy may be made for personal use only. Systematic electronic or print reproduction and distribution, duplication of any material in this paper for a fee or for commercial purposes, or modification of the content of the paper are prohibited. 


\section{TLPG FABRICATION SYSTEM}

The TLPG fabrication system used is shown in figure 1. The output from a Synrad $48-2 \mathrm{CO}_{2}$ laser with an output power of $5 \mathrm{~W}$ was used to heat the fibre (FibreCore SM750, cut off wavelength $630 \mathrm{~nm}$ ) to its melting point and allow tapers to be formed. The laser was cooled with an external water chiller at a temperature of $18.0 \pm 0.1{ }^{\circ} \mathrm{C}$, ensuring the laser power stability was $\pm 2.75 \%$. ZnSe optical elements were used for power tapping and injecting the HeNe alignment laser into the path of the $\mathrm{CO}_{2}$ laser beam. A ZnSe plano-convex cylindrical lens with a focal length of $100 \mathrm{~mm}$ was used to produce an elliptical focused spot on the fibre. The minor axis of the ellipse was $355 \pm 30 \mu \mathrm{m}$ in length and was parallel with the fibre axis, while the major axis was $3.5 \pm 0.03 \mathrm{~mm}$ and perpendicular to the fibre axis.

Two programmable rotation stages (Physik Instrumente) supported and located the fibre to be heated and stretched. The capstans mounted on the stages were inscribed with v-grooves to ensure reproducible fibre placement in the focal plane of the $\mathrm{ZnSe}$ lens. The fibre was attached to the stages using adhesive tape, with a $6 \mathrm{~g}$ weight used to pre-tension the fibre during the attachment process. This was found to reduce the variability in the diameter of the first taper compared to the subsequent tapers produced later in the process.

The operation of the laser and rotation stages were controlled using a program written in LabVIEW, which provided the requisite driving waveforms to control the laser power and the movement of the rotation stages. In addition, the program provided the necessary synchronization between the process steps, such that the process was repeatable. To fabricate a taper, the system was programmed such the output power of the laser was raised to $5 \mathrm{~W}$ and the stages pulled the fibre in opposite directions with a tangential velocity of $6 \mu \mathrm{m} \mathrm{s}^{-1}$ for a user defined duration. The laser power was then reduced to zero and the rotation of the stages stopped. The taper length was determined using the angular data provided by the rotation stage driver. The fibre was then translated by moving both rotation stages in the same direction to arrive at the position for the formation of the next taper. This sequence of stretching and translating was repeated until the requisite number of tapers was formed.

To optimize and to ensure the repeatability of the TLPG fabrication process, the following monitoring techniques were used $^{13}$; a NIR camera (Vosskuhler NIR-300P) was used to aid the alignment of the heated fibre within the focused laser spot and to observe the thermal intensity distribution along the fibre; a CCD spectrophotometer (Ocean Optics) and a tungsten-halogen light source were used to facilitate the measurement of the transmission spectrum of the TLPG over the wavelength range $625-1100 \mathrm{~nm}$ during the fabrication process.

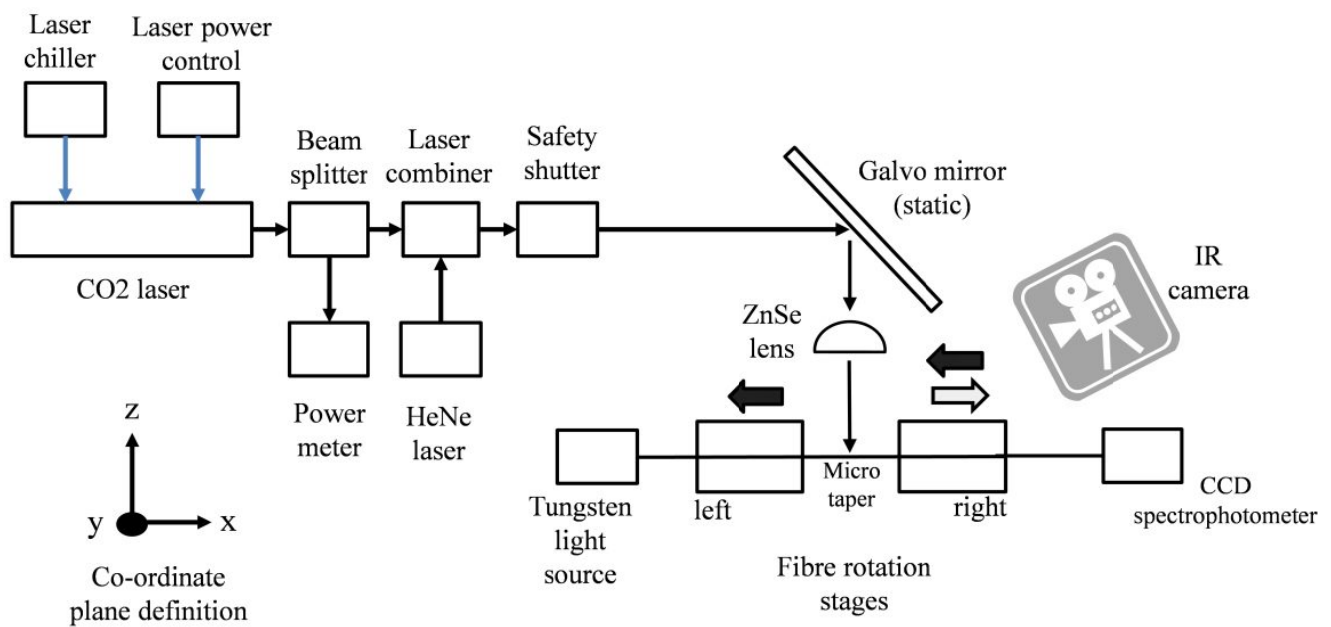

Figure 1. Schematic of the TLPG fabrication system. When a taper is formed the stages rotate in opposite directions. During fibre translation the stages rotate in the same direction. 


\section{EXPERIMENTAL RESULTS AND DISCUSSION}

Early TLPGs produced by this system exhibited high transmission losses ( $>15 \mathrm{~dB})$ and distorted resonance bands. The likely causes of this inferior performance were identified as: poor laser stability, a distorted first taper, variable fibre pretensioning, unoptimised taper waist diameter and variations in grating period along the length of the LPG. The laser stability was improved to $\pm 2.75 \%$ by using a high performance water cooling chiller and ensuring the laboratory air temperature was controlled to $\pm 1 \%$. Initial experiments revealed considerable variation between the transmission spectra of the fabricated TLPGs. This was found to arise primarily from inconsistencies in the characteristics of the first taper in each LPG, cause by variations in the tension applied to the fibre at the start of the fabrication process and the influence of twisting of the fibre when positioning it in the fabrication rig. To reduce the distortion of the first taper, the fibre was fixed to the stages with minimal rotational twist and a $6 \mathrm{~g}$ weight was used to ensure that the initial fibre tensioning was reproducible.

To improve the insertion loss of the TLPGs produced, a number of experiments were undertaken to investigate the influence of the diameter of the taper waist and the taper half-angle. Various taper pull times were trialed, ranging from $13 \mathrm{~s}$ to $18 \mathrm{~s}$. The use of a pull-time of $15 \mathrm{~s}$, which produced a waist diameter of $117 \mu \mathrm{m}$, resulted in the lowest insertion loss. Using a taper pull-time of $15 \mathrm{~s}$, a TLPG consisting of 6 tapers was fabricated. The spectral performance is illustrated in figure 2 . The average taper waist diameter was $117 \pm 2 \mu \mathrm{m}$, the average period spacing was $418 \pm 11 \mu \mathrm{m}$ and the duty cycle was $54 \pm 6 \%$. The passband transmission at $825 \mathrm{~nm}$ is $\sim 57 \%$ and does not substantially degrade when successive tapers were fabricated.

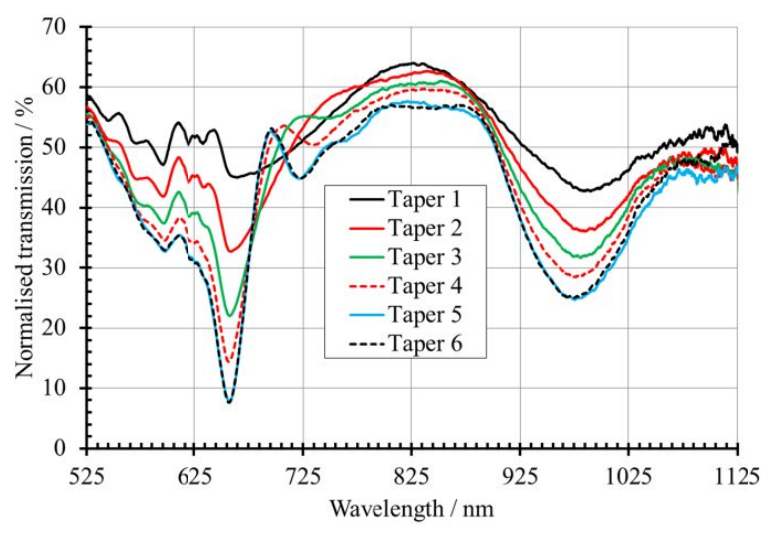

Figure 2: The evolution of the transmission spectrum of a TLPG consisting of 6 micro-tapers with waist diameters of $117 \mu \mathrm{m}$ and a period of $418 \mu \mathrm{m}$, using SM750 fibre.

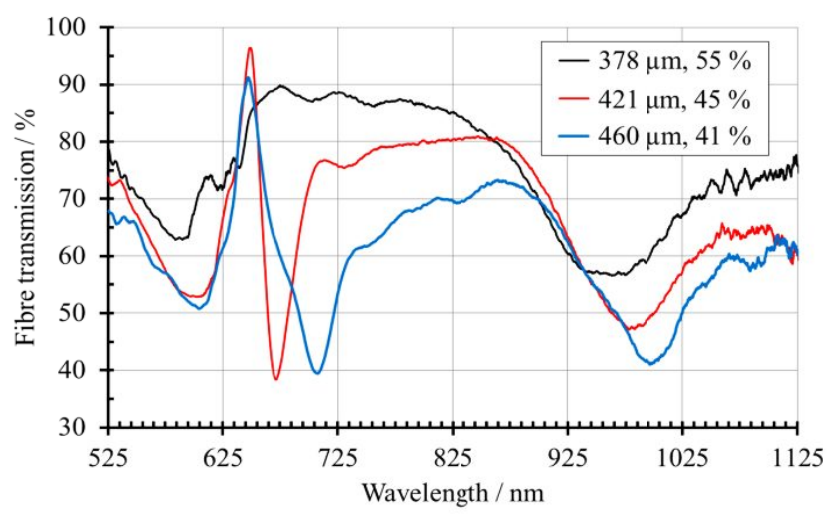

Figure 3: Transmission of differing 6 micro-taper TLPGs with periods of 378,421 and $460 \mu \mathrm{m}$ and duty cycles of 55,45 and $41 \%$. The fibre used was SM750.

While the duty cycle of this TPLG was measured at $54 \%$, the target was $50 \%$. It is known from studies of UV-written LPGs, that duty cycles near 50\% can produce high transmission and increased spectral resonance band definition ${ }^{12}$. The estimation of percentage duty-cycle is subject to significant error $( \pm 6 \%)$ as it was not possible to determine the contribution of laser induced refractive index modulation of the fibre, so only the geometrical modulation was assessed by measuring the total taper length, using an Olympus BX51 microscope.

To examine the effect of changes in duty-cycle on the spectrum of the TLPGs, three TLPGs were fabricated. Each TLPG comprised of 6 tapers and the duty-cycle was varied from $41-55 \%$. The spectral performance of the differing TLPGs is shown in figure 3 . As the grating period increased, the central wavelength of the resonance bands increased, as expected. The transmission level in the pass-band region $(\sim 775 \mathrm{~nm})$ is influenced by the duty-cycle value, with a duty-cycle of $55 \%$ resulting in a reduced pass-band optical loss of $0.6 \mathrm{~dB}$. This TLPG has a grating period of $378 \mu \mathrm{m}$ and a length of only $2.27 \mathrm{~mm}$, which is substantially shorter than conventional UV-written gratings which can be $30 \mathrm{~mm}$ long ${ }^{3}$. 


\section{CONCLUSIONS}

The process of fabricating TLPGs using a $\mathrm{CO}_{2}$ laser is described. These were assessed over the spectral waveband: 625$1100 \mathrm{~nm}$. By optimization of the taper waist diameter and careful control of the duty-cycle and its uniformity along the length of the grating, TLPGs of period spacings $\geq 378 \mu \mathrm{m}$, consisting of 6 periods with a corresponding total physical length of $2.27 \mathrm{~mm}$, were fabricated. These exhibited an insertion loss of $0.6 \mathrm{~dB}$ and had resonance band extinction values of $3 \mathrm{~dB}$.

\section{ACKNOWLEDGEMENTS}

The work was supported by funding from the Engineering and Physical Sciences Research Council (EPSRC) UK (EPL010437, EP-H02252X). Data underlying this study can be accessed through the Cranfield University repository at http://dx.doi.org/10.17862/cranfield.rd.3969153.

\section{REFERENCES}

1. Vengsarkar, A. M., Lemaire, P. J., Judkins J. B., Bhatia V., Erdogan T. and Sipe J. E., "Long period fiber gratings as band-rejection filters," J. Light. Technology, 14, 58-65 (1996).

2. Peterka, P., Maria, J. and Dussardier, B., "Long-period fiber grating as wavelength selective element in double-clad Yb-doped fiber-ring lasers," Laser Phys. Lett., 6, 732-736 (2009).

3. James, S. W. and Tatam, R. P., "Optical fibre long-period grating sensors : characteristics and application," Meas. Sci. Technol., 14, R49-R61 (2003).

4. Wang, Y., "Review of long period fiber gratings written by $\mathrm{CO}_{2}$ laser," J. Appl. Phys., 108, 081101 (2010).

5. Yin, G., Wang, Y., Liao, C., Zhou, J., Zhong, X., Wang, G., Sun, B. and He, J., "Long period fiber gratings inscribed by periodically tapering a fiber," IEEE Photon. Technol. Lett., 26, 698-701 (2014).

6. Shao, L.-Y., Zhao, J., Dong, X., Tam, H. Y., Lu, C. and He, S., "Long-period grating fabricated using resistive filament heating", 19th International conference on optical fibre sensors, Proc. SPIE, Perth, Australia, 2008, 7004, $1-4$.

7. Castro Alves, D., Coelho, J. M. P., Nespereira, M., Monteiro, F., Abreu, M. and Rebordão, J. M., "Automation methodology for the development of LPFG using $\mathrm{CO}_{2}$ laser radiation", 8th Ibero-american Optics Meeting and 11th Latin American Meeting on Optics, Lasers and Applications, Proc. SPIE, 8785, 87854X 1-5 (2013).

8. Dianov, E. M., Karpov, K., Grekov, M. V., Golant, K. M., Vasiliev, S. A., Medvedkov, O. I. and Khrapko, R. R., "Thermo-induced long-period fibre gratings," European conference on Optical Communications, Edinburgh, UK, 53-56 (1997) .

9. Kritzinger, R., Schmieder D. and Booysen, A., "Azimuthally symmetric long-period fibre grating fabrication with a $\mathrm{TEM}_{01}$ mode $\mathrm{CO}_{2}$ laser," Meas. Sci. Technol., 20, 1-8 (2009).

10. Liu, X., Yan, M., Zhan, L., Luo, S., Zhang Z. and Xia, Y., "Controlling of symmetric and asymmetric mode coupling in long-period fiber gratings single-side induced by long-pulse $\mathrm{CO}_{2}$ laser," Opt. Commun., 284, 12321237 (2011).

11. Smietana, M., Bock, W. J., Mikulic P. and Chen, J., "Increasing sensitivity of arc-induced long-period gratingspushing the fabrication technique toward its limits," Meas. Sci. Technol., 22,1-6 (2011).

12. Wong, R. Y. N., Chehura, E., Staines, S. E., James S. W. and Tatam, R. P., "Fabrication considerations for fiber optic long period gratings operating at the phase matching turning point," Appl. Opt., 53, 4669-4674 (2014).

13. Mullaney, K., Correia, R., Staines, S., James S.W. and Tatam, R. P., "Monitoring techniques for the manufacture of tapered optical fibres", Appl. Opt., 54, 8531-8536 (2015). 
2017-04-23

\section{Optimised process for fabricating tapered long period gratings}

Mullaney, Kevin

SPIE

K. Mullaney; S.E. Staines; S.W. James; R.P. Tatam, Optimised process for fabricating tapered long period gratings, 25th International Conference on Optical Fiber Sensors, 2017, Jeju,

Republic of Korea.

http://dx.doi.org/10.1117/12.2263275

Downloaded from Cranfield Library Services E-Repository 